\title{
The measurement of temporal changes in plasma glucose concentration and energy consumption with respiratory and metabolism analysis system for animals and continuous glucose monitoring system
}

\author{
Shinichi Ozato, Seichi Katayama, Naoyuki Hironaka, Katsuhide Nishi
}

Kumamoto Laboratory, LSI Medience Corporation, Japan

In this study, the temporal respiratory metabolism was measured with the respiratory and metabolism analysis system for animals (Arco system Inc.) in mice. At the time, the device which could be measured the food consumption and the locomotor activity (Shinfactory co., Ltd.) and the continuous glucose monitoring system (Primtech corporation) were combined with the respiratory and metabolism analysis system. By using this system, the temporal changes in plasma glucose concentration and energy consumption in mice were measured at the same time. The mice at 8 and 36-week of age were used in order to verify the influence with age. In the measurement, feed restrictions for 2 hours (1 hour immediately after the dark period and 1 hour just before the light period) were implemented. Result, the change of the glucose concentration associated with the food intake and that of the breathing quotient was able to be detected. Moreover, the difference of the plasma glucose concentration change due to aging could be also detected. The results suggested that this system was able to be measured the energy metabolism with high sensitivity. 